\title{
$\left[{ }^{18} \mathrm{~F}\right] \mathrm{FMCH}$ PET/CT biomarkers and similarity analysis to refine the definition of oligometastatic prostate cancer
}

\author{
Martina Sollini ${ }^{1,2}$, Francesco Bartoli ${ }^{3}$, Lara Cavinato ${ }^{4}$, Francesca leva ${ }^{4,5}$, Alessandra Ragni ${ }^{4}$, Andrea Marciano ${ }^{3}$, \\ Roberta Zanca ${ }^{3}$, Luca Galli ${ }^{6}$, Fabiola Paiar ${ }^{7}$, Francesco Pasqualetti ${ }^{7}$ and Paola Anna Erba ${ }^{3,8,9^{*}}$ (D)
}

\begin{abstract}
Background: The role of image-derived biomarkers in recurrent oligometastatic Prostate Cancer (PCa) is unexplored. This paper aimed to evaluate $\left.{ }^{18} \mathrm{~F}\right] \mathrm{FMCH}$ PET/CT radiomic analysis in patients with recurrent PCa after primary radical therapy. Specifically, we tested intra-patient lesions similarity in oligometastatic and plurimetastatic PCa, comparing the two most used definitions of oligometastatic disease.

Methods: $\mathrm{PCa}$ patients eligible for $\left[{ }^{18} \mathrm{~F}\right] \mathrm{FMCH}$ PET/CT presenting biochemical failure after first-line curative treatments were invited to participate in this prospective observational trial. PET/CT images of 92 patients were visually and quantitatively analyzed. Each patient was classified as oligometastatic or plurimetastatic according to the total number of detected lesions (up to 3 and up to 5 or $>3$ and $>5$, respectively). Univariate and intra-patient lesions' similarity analysis were performed.
\end{abstract}

Results: $\left[{ }^{18} \mathrm{~F}\right] \mathrm{FMCH}$ PET/CT identified 370 lesions, anatomically classified as regional lymph nodes and distant metastases. Thirty-eight and 54 patients were designed oligometastatic and plurimetastatic, respectively, using a 3-lesion threshold. The number of oligometastic scaled up to 60 patients (thus 32 plurimetastatic patients) with a 5-lesion threshold. Similarity analysis showed high lesions' heterogeneity. Grouping patients according to the number of metastases, patients with oligometastatic PCa defined with a 5-lesion threshold presented lesions heterogeneity comparable to plurimetastic patients. Lesions within patients having a limited tumor burden as defined by three lesions were characterized by less heterogeneity.

Conclusions: We found a comparable heterogeneity between patients with up to five lesions and plurimetastic patients, while patients with up to three lesions were less heterogeneous than plurimetastatic patients, featuring different cells phenotypes in the two groups. Our results supported the use of a 3-lesion threshold to define oligometastatic PCa.

Keywords: $\left[{ }^{18} \mathrm{~F}\right] \mathrm{FMCH}$ PET/CT, Epithelial-mesenchymal transition, Number of lesions, Oligometastatic PCa, Radiomics, Silhouette index, Similarity analysis, Biomarkers

*Correspondence: p.erba@unipi.it

${ }^{3}$ Nuclear Medicine, Department of Translational Research and Advanced Technology in Medicine and Surgery University of Pisa, Pisa University Hospital, Via Roma 67, 56123 Pisa, Italy

Full list of author information is available at the end of the article

\section{Introduction}

${ }^{18} \mathrm{~F}$-fluoro-methyl-choline $\left(\left[{ }^{18} \mathrm{~F}\right] \mathrm{FMCH}\right)$ or ${ }^{11} \mathrm{C}$-choline positron emission tomography/computed tomography $(\mathrm{PET} / \mathrm{CT})$ is a well-established imaging modality to detect recurrence in Prostate Cancer $(\mathrm{PCa})$ patients with biochemical failure after definitive treatment. About 30\% of patients radically treated will recur [1], and the early 
diagnosis of disease relapse is pivotal to guide treatment decision [2-4]. The extensive use of functional imaging resulted in the identification of a new clinical disease entity namely oligometastatic disease (i.e., limited tumor burden) $[5,6]$.The theory of oligometastatic disease dates back to 1995 [7]. The interest in oligometastatic disease relies on biological features and clinical evidences resulting in better outcomes compared to plurimetastatic disease [8]. Consequently, the paradigm of metastatic $\mathrm{PCa}$ has evolved and, nowadays, tumor burden is an indicator for treatment allocation [5, 9-13]. The concept of oligometastatic disease is evolving by moving from a definition purely based on the number of lesions and/or involved organs to the identification of key features capable to biologically describe oligometastatic (and multimetastatic) phenotype to assign a patient to local or systemic treatment [14]. Quantitative imaging analysis can provide a number of biomarkers to be used in this setting. Indeed, advances in quantitative methods for data analysis have widened the scope of what imaging as a technology can enable in terms of scientific discovery. During the latest years, imaging data analysis have progressively shifted from a qualitative-semantic descriptors to quantitative measurements extracted by high-throughput methods able to make medical data mineable, namely radiomics. Accordingly, analysis of surface or volume, traditionally described by texture, has evolved in a discipline (in between image analysis and statistical modeling) aiming at describing tumor lesion heterogeneity through a multitude of quantitative indices (i.e., radiomic features). Hence, it's possible to capture information about the relations between pixels or voxels of a region or a volume of interest, by applying post-processing formulas to medical images acquired within the routinely clinical workflow of patients. Radiomic features can be extracted from any type of images from pictures, to pathology images, to cross-sectional images, to molecular imaging, to hybrid imaging $[15,16]$. Although molecular imaging is largely used to define tumor burden and successfully select oligometastatic patients eligible for local treatment $[17,18]$, the role of image-derived biomarkers in oligometastatic PCa remains to be determined.

The present work aimed to evaluate the feasibility of radiomic analysis of $\left[{ }^{18} \mathrm{~F}\right] \mathrm{FMCH} \mathrm{PET} / \mathrm{CT}$ in patients with recurrent $\mathrm{PCa}$ after primary radical therapy. The goal of this research is two-fold. First, we investigated radiomic features extracted from $\left[{ }^{18} \mathrm{~F}\right] \mathrm{FMCH}$ PET/CT images according to the site of recurrence and tumor burden. Second, we explored intra-patient lesions similarity to provide biologically insights into oligometastatic (and plurimetastatic) recurrent PCa. Eventually, this approach provides an integrated multidimensional definition of oligometastatic recurrent $\mathrm{PCa}$ comprising image-derived features, going beyond the mere concept of the tumor burden.

\section{Materials and methods \\ Study design and patient selection}

All PCa patients eligible for $\left[{ }^{18} \mathrm{~F}\right] \mathrm{FMCH} \mathrm{PET} / \mathrm{CT}$ presenting with biochemical failure after first-line curative treatments were invited to participate in this observational trial. Biochemical relapse was defined according to clinical guidelines [19]. This preliminary analysis consisted of 92 patients (mean age $72 \pm 7$ years, median age 72 years, range $55-85)$ with a positive $\left[{ }^{18} \mathrm{~F}\right] \mathrm{FMCH} \mathrm{PET} / \mathrm{CT}$ prospectively enrolled between January 2011 and February 2018 in the above-mentioned trial. Demographic and clinical data including age, Gleason score (GS) at diagnosis, prostate specific antigen (PSA) level at the time of $\left[{ }^{18} \mathrm{~F}\right] \mathrm{FMCH} \mathrm{PET} / \mathrm{CT}$, primary treatment, and androgen deprivation therapy (ADT; if yes, ongoing or discontinued) were collected for all patients. Patients baseline characteristics are summarized in Table 1.

\section{$\mathrm{PET} / \mathrm{CT}$ image acquisition and analysis}

$\left[{ }^{18} \mathrm{~F}\right] \mathrm{FMCH}$ PET/CT were acquired at Regional Center of Nuclear Medicine of the Azienda Ospedaliero-Universitaria Pisana with an integrated PET/CT system General Electric Discovery 710 (General Electric Healthcare, Waukesha, WI, USA). Image acquisition was performed according to version 1.0 [20] and version 2.0 [21] of the European Association of Nuclear Medicine (EANM) guidelines until and from February 2015, respectively. Image acquisition protocol is detailed in Additional file.

$\mathrm{PET} / \mathrm{CT}$ images were visually interpreted by two experienced nuclear medicine physicians (PAE and AM), aware of the patient's medical history. Lesions were anatomically designed as regional or distant metastases according to the TNM staging system [22]. Each patient was defined as oligometastatic or plurimetastatic based on $\left[{ }^{18} \mathrm{~F}\right] \mathrm{FMCH}$ PET/CT findings. We arbitrary decided to use the two most frequently applied definitions of oligometastatic $\mathrm{PCa}$, and classify patients twice based on the number of lesions. Accordingly, oligometastic disease was defined as PCa patients having with up to three [23] and up to five lesions [24]. Lesions were semiautomatically segmented by the PET VCAR software (GE Healthcare, Waukesha, WI, USA) on a General Electric workstation. A volume of interest (VOI) was draw for each lesion and visualized on CT images to check the anatomical correspondence. Radiomic features $(n=42)$ were extracted from each VOI by using the LIFEx software [25] (http://www.lifexsoft.org). Image processing and calculation of image-derived features are reported in Additional file 1: Table S1, according to the IBSI reporting guidelines [26]. 
Table 1 Baseline characteristics

\begin{tabular}{|c|c|c|}
\hline Variable & Mean (median) & Range (std) \\
\hline Age & $72.09(71.68)$ & $54.88-85.24(7.03)$ \\
\hline Total volume (mL) & $16.41(3.16)$ & $0.22-207.70(34.72)$ \\
\hline Gleason score & $7.37(7.0)$ & $5.0-9.0(1.027)$ \\
\hline PSA & $18.16(2.66)$ & $0.09-591.0(70.96)$ \\
\hline Variable & & Number (\%) \\
\hline \multicolumn{3}{|l|}{ Number of metastases } \\
\hline Oligometastatic $(<3)$ & & $38(41.30)$ \\
\hline Plurimetastatic ( $\geq 3$ ) & & $54(58.70)$ \\
\hline Oligometastatic $(<5)$ & & $60(65.22)$ \\
\hline Plurimetastatic ( $\geq 5$ ) & & $32(34.78)$ \\
\hline Oligometastatic $(3 \leq n<5)$ & & $22(23.92)$ \\
\hline \multicolumn{3}{|l|}{ Gleason category } \\
\hline Gleason $(\leq 7)$ & & $53(63.10)$ \\
\hline Gleason (>7) & & $31(36.90)$ \\
\hline \multicolumn{3}{|l|}{ Ongoing therapy } \\
\hline YES & & $33(35.87)$ \\
\hline NO & & $59(64.13)$ \\
\hline \multicolumn{3}{|l|}{ Initial therapy } \\
\hline $\mathrm{RP}$ & & $23(25.0)$ \\
\hline$R P+R T$ & & $52(56.52)$ \\
\hline RT & & $9(9.78)$ \\
\hline
\end{tabular}

\section{Statistical analysis}

Patient characteristics were summarized in frequency tables, and descriptive statistics was provided.

Univariate analysis was used to assess the discriminant power of radiomic features, according to pre-specified clinical categories belonging to patients (i.e., oligometastatic versus plurimetastatic, $G S \leq 7$ versus $G S>7$ ) and lesions description (regional lymph nodes versus distant lymph nodes, and regional or distant lymph nodes versus bone metastases). The Mann-Whitney test (two-tailed) was used to evaluate marginal significance of each radiomic variable in the considered population. To capture the potential impact of ongoing ADT on textural features, such analysis was performed considering i) all patients, ii) the subset of patients undergoing ADT therapy, and iii) the subset of patients not on ADT. Significance has been confirmed with $p \leq 0.01$ for GS and $p \leq 0.001$ for all the other clinical categories.

Furthermore, PSA was considered apart from radiomic variables. PSA distribution in different groups was compared slicing patients according to pre-specified clinical categories (i.e., oligometastatic versus plurimetastatic, GS $\leq 7$ versus $G S>7$ ). Tests were performed on PSA values, and patients belonging to different groups were compared in terms of PSA distribution. The entire population of patients and the patients undergoing or not ADT were considered separately. PSA significance has been confirmed with $p \leq 00.05$, and results were discussed accordingly. Analyses were carried-out on SPSS version 25.

Additionally, a similarity analysis aimed at explored intra-patient lesions heterogeneity was performed as detailed in Additional file 1. Intra-patient lesions' similarity was assessed using the silhouette index (i.e., similarity index) [27]. The silhouette index ranges from -1 to 1. A value close to 1 entails a high intra-patient lesions' similarity. Conversely, a negative silhouette index indicates a low intra-patient lesions' similarity, with proximity or even overlap of lesions belonging to different patients in the latent space. For all those patients with just one lesion, the silhouette is set to zero and taken apart from any analysis. Similarity analysis included patient-based analysis, anatomy-based analysis, and metabolism-based analysis.

Kruskal test was used to compare intra-patient all-lesions' similarity in different groups obtained splitting patients according to pre-specified clinical categories (i.e., oligometastatic/plurimetastatic, Gleason Score $\leq 7 />7$, median PSA in the population at the time of PET/CT $>1.93 \mathrm{ng} / \mathrm{mL} / \leq 1.93 \mathrm{ng} / \mathrm{mL}$, and ongoing ADT treatment yes/no). 
Unsupervised clustering was used as final step to support intra-patient lesions' similarity analysis interpretation. Radiomic-based clusters characterization was explored in order to check if any prevalence of the categories previously described could be appreciated. Analyses were carried-out on Python.

\section{Results}

$\left[{ }^{18}\right.$ F]FMCH PET/CT identified 370 lesions, anatomically classified as regional lymph nodes $(n=68)$ and distant metastases $(n=302)$. Distant metastases included distant lymph nodes $(n=81)$ and bone lesions $(n=221)$ (Additional file 1: Table S2). Using a cut-off of three lesions to define a limited tumor burden, 38 patients were oligometastatic and 54 plurimetastatic (Fig. 1). The number of oligometastic scaled up to 60 patients (thus 32 plurimetastatic patients) with a 5 lesions threshold (Fig. 1).

\section{Exploratory analysis}

A number of features emerged as significant at univariate analysis (Additional file 1: Table S3) when considering tumor burden, GS, and distant or regional lymph nodes versus bone metastases. A significant trend toward a progressive reduction of the number of features was present moving form whole population, to the subset of patients under ADT to patients ADT-off.

Patients with plurimetastatic disease exhibited higher PSA values than patients with limited disease burden (Table 2), irrespectively of the criterion used for defining oligometastatic disease (up to 3 or 5 lesions), with

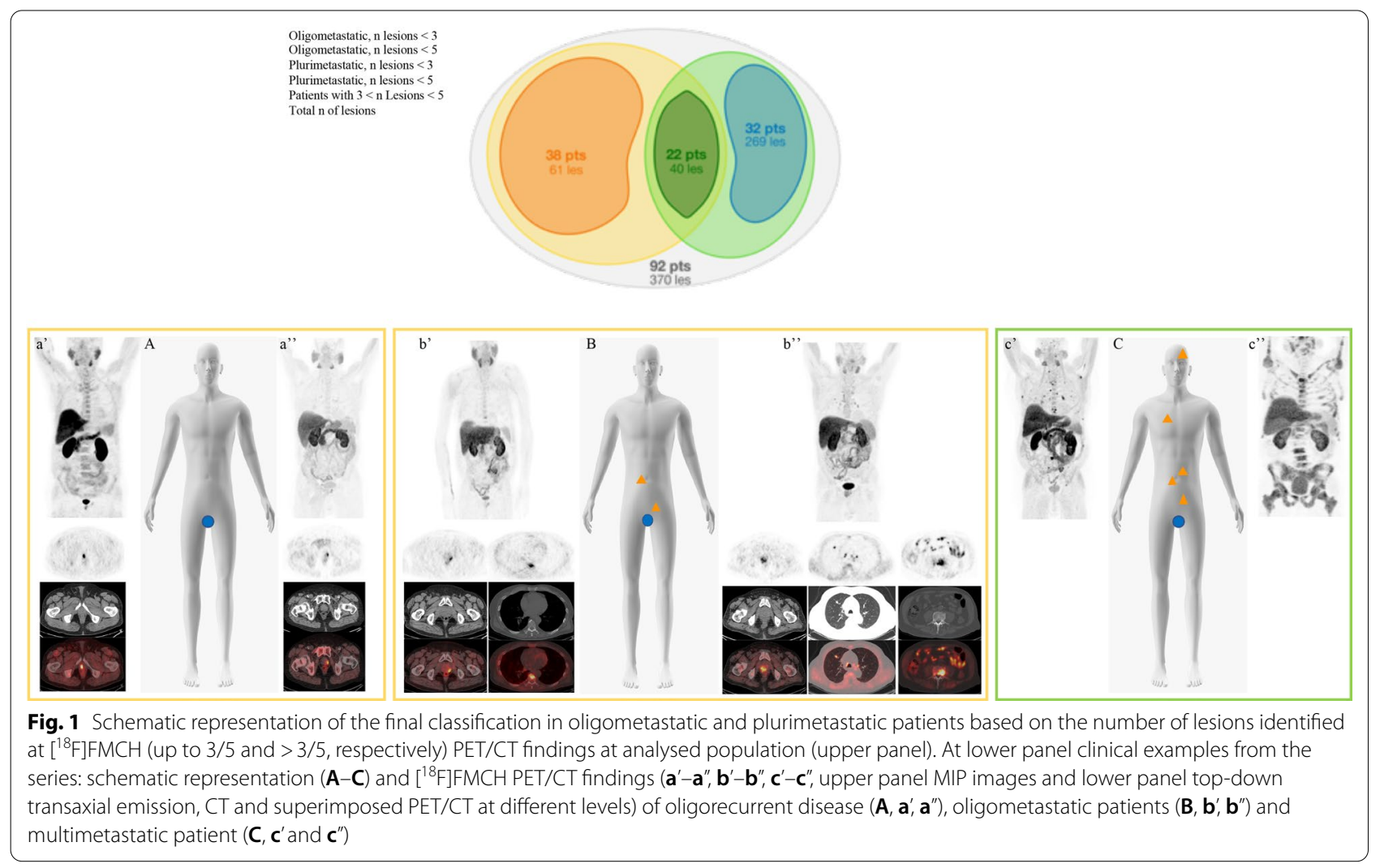

Table 2 Values of PSA at the time of PET/CT based on tumor burden

\begin{tabular}{|c|c|c|c|c|c|c|}
\hline PSA & Oligometastatic $\leq \mathbf{3}$ & Multimetastatic $>3$ & $p$-value & Oligometastatic $\leq 5$ & Multimetastatic $>5$ & $p$-value \\
\hline Mean & 3.6959574 & 38.162941 & 0.0015 & 3.524 & 59.99095 & $<.0001$ \\
\hline Dev std & 5.3415206 & 107.03347 & & 4.939003 & 132.6206 & \\
\hline Min & 0.09 & 0.24 & & 0.09 & 1.93 & \\
\hline Max & 32 & 591 & & 32 & 591 & \\
\hline Median & 1.85 & 4.73 & & 1.8 & 13.05 & \\
\hline
\end{tabular}


a higher correlation using the 5 lesions threshold. PSA values also differed significantly between plurimetastatic disease with more than 5 lesions and patients with more than 3 and not more than 5 lesions $(p$-value $=0.001)$. In addition, PSA values were higher in patients with bone recurrence as compared to the ones with regional lymph nodes metastasis ( $p$-value $<0.0001$; Table 3 ). No difference in PSA levels were found in patients with a $\mathrm{GS} \leq 7$ or $>7(p=1)$ or in patients ADT-on or ATD-off $(p=0.07)$.

\section{Similarity analysis}

$\left[{ }^{18} \mathrm{~F}\right] \mathrm{FMCH}$ PET/CT identified multi-lesions disease in 55 patients. Patient-based analysis results are shown in Fig. 2. The mean similarity index was -0.462 , indicating that lesions were poorly matched to the ones belonging to the same patient, and much more closed to other patients' lesions. Anatomy-based analysis results are shown in Additional file 1: Fig. S1. The mean similarity index was negative, regardless of the site of recurrence $(-0.291$ for regional lymph nodes, -0.343 for distant lymph nodes, and -0.429 for bone metastases). At the paired $t$-test, the mean anatomy-based similarity index was significantly different from the patient-based similarity index in all three cases $(p<0.01)$. Organ-specific lesions lead to a reduced intra-patient heterogeneity with respect to all the lesions. The mean similarity index of the metabolism-based analysis (Additional file 1: Fig. S2) was negative $(-0.380$ for the first tertile, -0.380 for the second tertile, and -0.428 for the third tertile), although showing lower heterogeneity when considering only the maximum standardized uptake value $\left(\mathrm{SUV}_{\max }\right)$-wise homogeneous lesions for computing patients' silhouette. At the paired t-test significant differences between mean similarity index were found $(p$ $<0.01$ ), suggesting quite a concordance between higher radiomic features and $\mathrm{SUV}_{\max }$ statistical parameter. Patients' lesions result into a more homogeneous clustering when sliced per $S U V_{\text {max }}$ tertile while show higher heterogeneity when considered all together.

Grouping patients according to the number of metastases, the 3-lesion threshold definition of oligometastatic exhibited lower heterogeneity than plurimetastic disease (Fig. 3a; $p=0.0003$ ). The 5-lesions-threshold definition of oligometastatic disease showed a similar heterogeneity of plurimetastic disease (Fig. 3b; $p=1.337 \mathrm{e}-08$ ). These findings were also confirmed in patients classified with

Table 3 Values of PSA at the time of PET/CT based on site of recurrence

\begin{tabular}{llllll}
\hline PSA & Skeleton & Regional Ln & Distant Ln & p-value S-R & $\boldsymbol{p}$-value S-D \\
\hline Mean & 67.076039 & 9.3731667 & 18.236806 & $<0.001$ & 0.2291 \\
Dev std & 148.03796 & 23.508696 & 43.819157 & \\
Min & 0.09 & 0.1 & 1.06 & \\
Max & 591 & 128 & 211 & \\
Median & 5.77 & 2.72 & 3.81 & \\
\hline
\end{tabular}
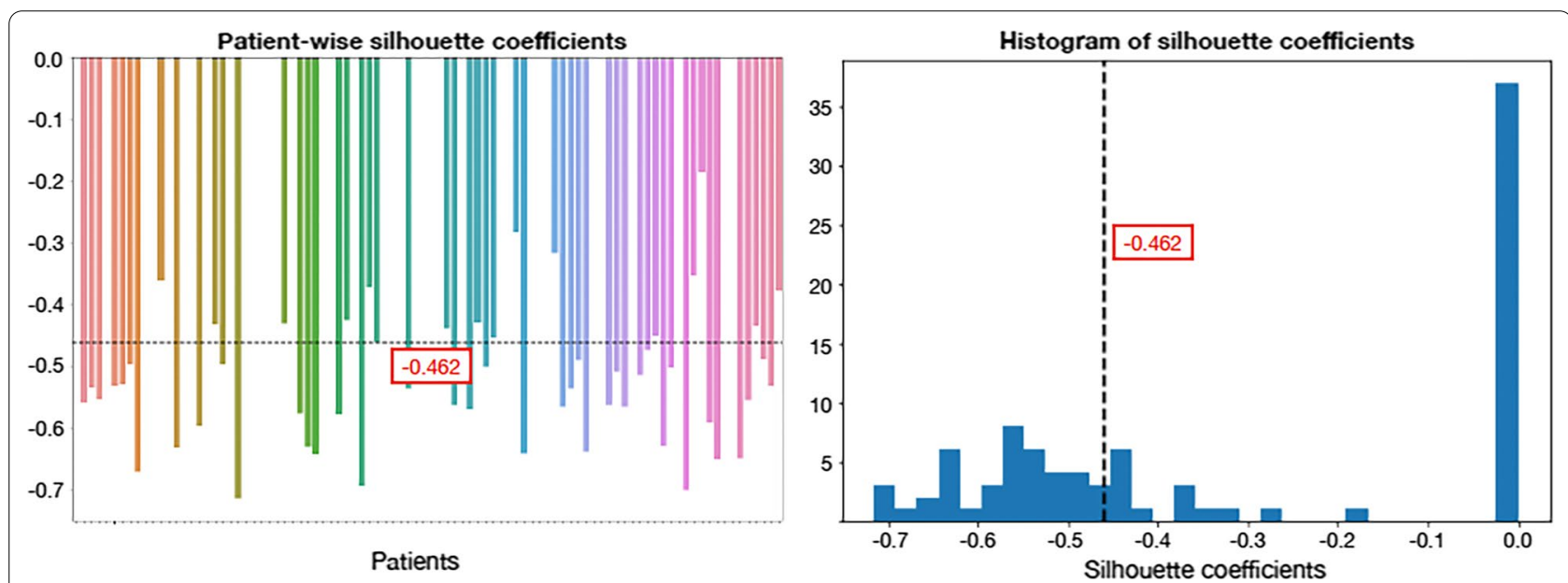

Fig. 2 Lesions' similarity within each patient. Patients were included in the plot with no specific sorting criterium. Subjects were indeed randomly attributed a number from 1 to 92 as to be univocally identified. This number was used as agnostic coding factor. Colors are also randomly attributed to patients according to a palette of our choice, such that each subject could be associated to both a number and a color for visualization purposes 


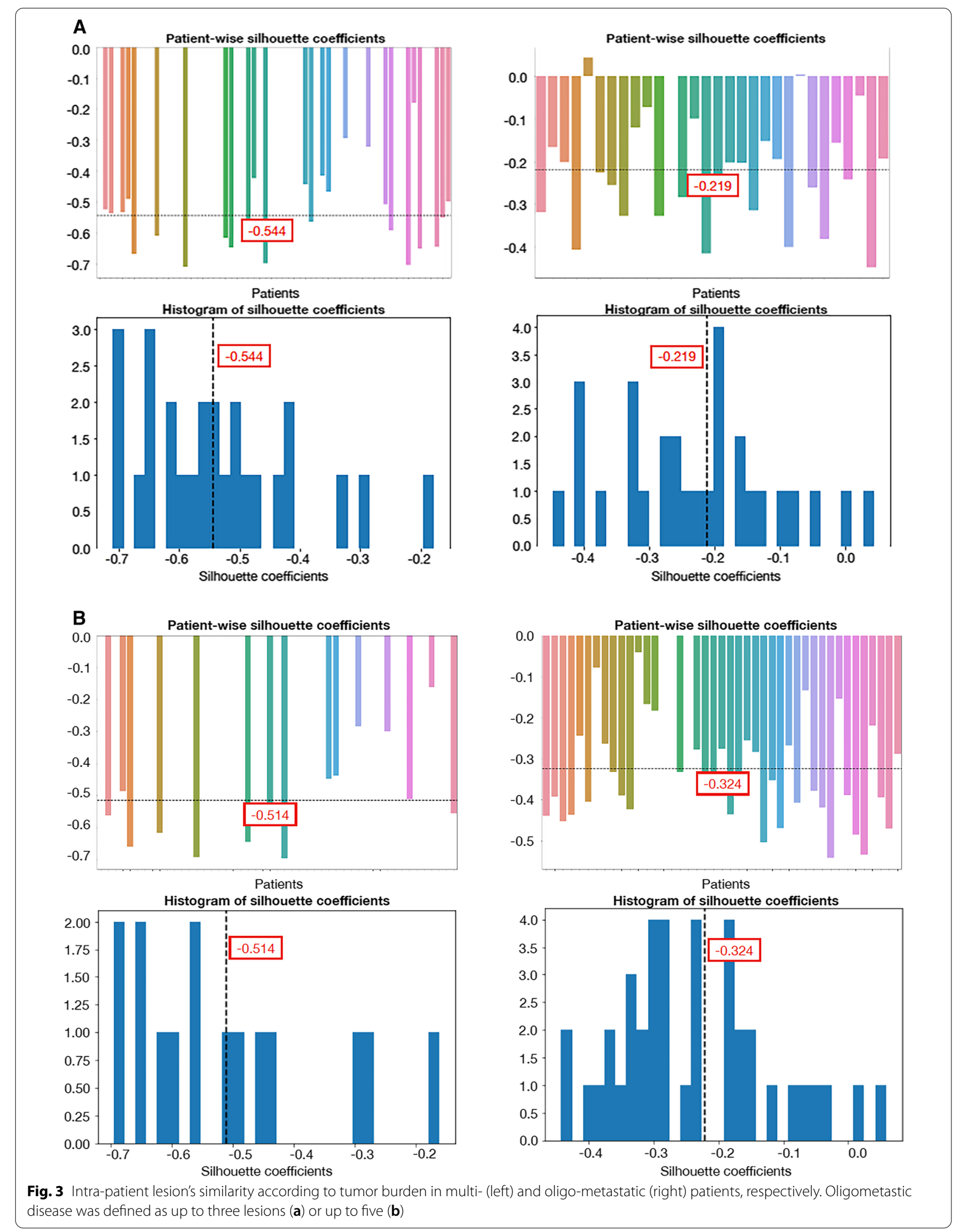


the three lesions threshold as compared to patients with a lesion number between three and five and with more than five lesions $(p=7.223 \mathrm{e}-06)$. Patients with oligometastatic disease with a maximum of three lesions were less heterogeneous than the other two groups. Intrapatient lesion's similarity was comparable when considering patients with a GS $\leq 7$ and $>7$ (Additional file 1: Fig. S3; $p=0.4068$ ), whereas low PSA patients exhibited lower yet not statistically different heterogeneity than patients with high PSA (Additional file 1: Fig. S4; $p=0.2723$ ). ADT weakly affected intra-patient lesion's similarity (Additional file 1: Figure S5; $p=0.0057$ ).

Unsupervised clustering identified two clusters as the best interpretable choice (Additional file 1: Figure S6). We found a higher prevalence of the $S U V_{\max }$ range 1 (i.e., first $S U V_{\max }$ tertile with respect to second and third tertile) in the second cluster, a higher prevalence of plurimetastatic disease (i.e., $>5$ lesions) in the first cluster, and a higher prevalence of bone metastases disease with respect to nodal metastases in the first cluster.

\section{Discussion}

$\mathrm{PCa}$ recurrence represents a biological complex setting including patients with oligorecurrence, oligometastatic single-organ or multi-organ disease as well as multi-organ plurimetastatic disease, this latter present in about $60 \%$ of our patients in this series. The setting of metastatic disease is still rather unexplored for radiomic analysis, and few data are available in literature. Among the open issues, we identified the lack of consensus on the number of lesions to be included in the radiomic analysis as well as the biological significance of different recurrent disease phenotypes i.e., multiple single-organ metastasis versus synchronous multi-organ metastasis. While radiomics in the setting of oligorecurrent disease is rather intuitive taking advantage from the workflow developed for primary tumor lesion, the approach to oligo-/plurimetastatic disease with a per lesion analysis is a pure simplistic model. Indeed, the presence of multiple metastasis in the same organ or the spread within different organs and/or parenchyma requires proper modeling in light of the biological complexity of each metastases. Consequently, an insightful patients' data transformation from "long" to "wide" format accounting for the relationship among all lesions is necessary. In this work, we performed an intra-patient similarity analysis exploring the silhouette index to quantify and assess such relationship. By this approach, we demonstrated the ability of $\left[{ }^{18} \mathrm{~F}\right]$ FMCH PET/CT radiomic analysis to differentiate disease recurrence site, predict the Gleason score, and tumor burden (oligometastatic versus plurimetastatic disease) in recurrent PCa patients. Specifically, our data showed the coexistence of multiple texture phenotypes within one single organ or in the same patient as demonstrated by the high lesions heterogeneity. Such heterogeneity significantly decrease when considering only lesions within the same organ rather than all the lesions globally, and when focusing on metabolically similar lesions characterized by comparable $S U V_{\max }$ values.

Additionally, our analysis suggested that oligometastatic disease should be limited to patients with no more than five lesions, with further benefit of a more conservative criterion of a maximum of three lesion threshold. In our series, patients with plurimetastatic disease exhibited higher PSA values than patients with a limited tumor burden, with a higher correlation when the five lesions threshold was used. This evidence was further supported by the presence of statistically different PSA values in the groups of patients with more than five lesions and patients with more than three-five lesions. Moreover, intra-patient lesions' similarity showed a relationship between PSA levels and intra-patient lesions' heterogeneity (the lower the PSA, the lower the intra-patient lesions' heterogeneity). In addition, the similarity analysis showed that patients with oligometastatic PCa defined with a 5-lesion threshold presented lesions heterogeneity comparable to plurimetastic patients. Differently, lesions within patients having a limited tumor burden as defined by three lesions were characterized by less heterogeneity, thus featuring different cells phenotypes. Indeed, the key metabolic nodes associated with tumor heterogeneity are the biological prerequisite for a different $\left[{ }^{18} \mathrm{~F}\right] \mathrm{FMCH}$ uptake, as result of enhanced entry of longchain fatty acids into the mitochondria or as precursors of eicosanoid metabolism, providing to oligometastatic and plurimetastatic disease (from low to high), either a highly proliferative phenotype or invasive epithelialmesenchymal-transition-like phenotypes, respectively, as shown by in vitro experiments [28-31]. Therefore, the similarity index provides a tool for a better insight of oligometastatic PCa, going beyond $\left[{ }^{18} \mathrm{~F}\right] \mathrm{FMCH} \mathrm{PET} / \mathrm{CT}$ visual image analysis numbering the lesions [8]. This is a fundamental step in the debate around the proper definition of oligometastatic $\mathrm{PCa}$ in the attempt of integrate image-derived phenotype information of the "biological oligometastatic concept".

We have to acknowledge some limitations. Firstly, the sample size was relatively small. However, all images were prospectively obtained and processed using a standardized acquisition protocol and the same radiomic workflow, making results consistent. Secondly, it should be bear in mind that the concept of oligometastatic (and plurimetastatic) disease may be affected by the imaging modality (including the tracer) and its spatial resolution as well as by the clinical setting (i.e., diagnosis, recurrence, or relapse), making any definition an 
approximation. On the other hand, the use of the gold standard to properly classify oligometastatic patients is unfeasible in daily practice. Therefore, although each definition-either based on clinical or imaging data-is burdened by the inherent limitation(s) of the used method, its refinement trough new insights results in a patient management improvement toward precision medicine. Moreover, the innate limit of imaging is the detection of macroscopic disease, and despite the understandable advantage of microscopic disease detection (e.g., circulating tumor DNA), the role of imaging is still crucial. Oligometastatic disease is almost a continuum of risk and could seem inappropriate to express a so complex biological entity with a number. However, as above-mentioned the most used approaches to define oligometastatic PCa rely mainly on numbers. Our study, evaluating lesions heterogeneity, suggested an evidence-based threshold for oligometastatic/plurimetastatic PCa.

Thirdly, the evaluation of heterogeneity of relatively small lesions-as are typically those of biochemical recurrent $\mathrm{PCa}-$ may seems useless. However, we equally manage "oligometastatic" patients with one regional nodal recurrence and with bone/visceral metastases, even if they are probably affected by biologically different diseases. This analysis demonstrated that image-derived biomarkers effectively entail more information that those "captured" by visual analysis, and that they may be used to "in-vivo" study some biological behaviors of the disease. Fourthly, radiomic features were extracted from $\left[{ }^{18} \mathrm{~F}\right] \mathrm{FMCH}$ which works quite well if PSA is greater than $2 \mathrm{ng} / \mathrm{mL}$, but which is known roughly half as sensitive as prostate-specific membrane antigen (PSMA). Therefore, although imaging shows early disease that is expected to become widespread, it cannot be distinguished from the biological significance of oligometastases [8]. Accordingly, we will expect that an even more restrictive threshold should be used to numerically namely oligometastatic PCa when using PSMA-based imaging.

Lastly, we evaluated tumor heterogeneity and intrapatients lesions' similarity without any correlation with patients outcome. However, it was out of the scope of the present work. Indeed, we make use of the radiomic features with the aim of quantitively identifying different $\mathrm{PCa}$ phenotypes within patients. We thus intend to provide a different perspective-and possibly a new vision-for imaging data exploitation for drawing insightful conclusion, under the "umbrella" of radiomics. Among the extensive, yet often inconclusive and methodologically lacking, literature that is emerging around radiomics-based machine learning models (i.e., machine learning methods applied to radiomic features extracted from images as is), we propose a sound statistical method for characterizing within-patient tumor heterogeneity.
Quality of methodology is an essential requirement for inclusion of new evidence emerged by from trials [32], especially in the developing field of advanced image analysis [33, 34]. Appropriateness of clinical definitions and strength of radiomic workflow (including data analysis) should be established before to assess any prognostic or predictive role of image-derived parameters, to be sure they will be meaningful.

\section{Conclusions}

$\left[{ }^{18} \mathrm{~F}\right] \mathrm{FMCH} \mathrm{PET} / \mathrm{CT}$ radiomic analyses provided invaluable information about tumor heterogeneity of PCa recurrence, entailing discriminant ability in differentiating the disease according to the site of recurrence and the tumor burden. Based on our model, the definition of oligometastatic PCa should include patients with no more than three lesions. Indeed, oligometastatic patients defined as having up to five lesions, exhibited a heterogeneity comparable to plurimetastic patients. Conversely, when the limited tumor burden was defined as more than three lesions, oligometastatic patients were less heterogeneous than plurimetastatic patients, featuring different cells phenotypes in the two groups. Such tumor heterogeneity has key metabolic nodes associated with high tumor $\left[{ }^{18} \mathrm{~F}\right] \mathrm{FMCH}$ uptake, highlighting the benefit of potential subpopulation-specific targets with important therapeutic implications by radiomics analysis.

\section{Abbreviations \\ $\left[{ }^{18} \mathrm{~F}\right] \mathrm{FMCH}:{ }^{18} \mathrm{~F}$-fluoro-methyl-choline; ADT: Androgen deprivation therapy; EANM: European Association of Nuclear Medicine; GS: Gleason score; SUV $_{\text {max }}$ Maximum standardized uptake value; PCa: Prostate cancer; PET/CT: Positron emission tomography/computed tomography; PSA: Prostate specific antigen; PSMA: Prostate-specific membrane antigen; VOI: Volume of interest.}

\section{Supplementary Information}

The online version contains supplementary material available at https://doi. org/10.1186/s13550-021-00858-8.

Additional file 1. Supplementary materials include a detailed description of image processing and calculation of image-derived features, methods used for similarity analysis, and results of visual analysis, univariate analysis and similarity analysis in specific groups of patients.

\section{Acknowledgements}

We acknowledge Dr. Riccardo Morganti of the Statistic Analysis Unit, Pisa University Hospital for his support in the explorative analysis, Prof. Duccio Volterrani, and all technologists and nurses of the Nuclear Medicine Department for the assistance during the PET/CT scans, Prof. Fabiola Paiar and Dr. Francesca Manassero for the assistance in data collection and patients management.

\section{Authors' contributions}

All the authors critically revised the paper and approved the submitted version of the manuscript. Specific contributions: Conception and design: PAE, FP. Imaging analysis and interpretation: PAE, MS. Data collection: PAE, AM, RZ, FB, $P F, L G$. Writing the article: MS, PAE, LC. Critical revision of the article: ALL. Final 
approval of the article: ALL. Statistical analysis: LC, Fl, AR (FI is responsible for statistical analysis). Data modeling: LC, FI. Obtained funding: PAE, FP. Overall responsibility: PAE. All authors read and approved the final manuscript.

\section{Funding}

This report was composed within a study funded by Bando AIRC IG 2017 Id. 20819 "Oligometastatic and Oligorecurrent Prostate Cancer: enhancing patients' selection by new imaging biomarkers".

\section{Availability of data and materials}

The manuscript represents valid work and neither this manuscript nor one with substantially similar content under the same authorship has been published or is being considered for publication elsewhere. Paola Anna Erba had full access to all the data in the study and takes responsibility for the integrity of the data and the accuracy of the data analysis. Raw data are available on specific request to the corresponding author.

\section{Declarations}

\section{Ethics approval and consent to participate}

All procedures performed in were in accordance with the ethical standards of the institutional and national research committee and with the 1964 Declaration of HELSINKI and its later amendments or comparable ethical standards. This observational trial was approved by the Ethics Committee of the Pisa University Hospital (Pisa 8424/2015).

\section{Consent for publication}

Informed consent for participation in the clinical study and data publication was obtained from all patients included in the study.

\section{Competing interests}

The authors declare that they have no competing interests.

\section{Author details}

'Department of Biomedical Sciences, Humanitas University, Via Rita Levi Montalcini 4, Pieve Emanuele, Milan, Italy. ${ }^{2}$ RCCS Humanitas Research Hospital, Rozzano, Milan, Italy. ${ }^{3}$ Nuclear Medicine, Department of Translational Research and Advanced Technology in Medicine and Surgery University of Pisa, Pisa University Hospital, Via Roma 67, 56123 Pisa, Italy. ${ }^{4}$ MOX - Modeling and Scientific Computing, Department of Mathematics, Politecnico di Milano, p.zza Leonardo da Vinci 32, 20133 Milan, Italy. ${ }^{5}$ CADS - Center for Analysis, Decision and Society, Human Technopole, Milan, Italy. ${ }^{6}$ Medical Oncology, Pisa University Hospital, Via Roma 67, 56123 Pisa, Italy. ${ }^{7}$ Radiation Oncology, Pisa University Hospital, Via Roma 67, 56123 Pisa, Italy. ${ }^{8}$ University Medical Center Groningen, Medical Imaging Center, University of Groningen, Groningen, The Netherlands. ${ }^{9}$ Department of Translational Research and New Technology in Medicine and Surgery, University of Pisa, Via Savi 10, 56126 Pisa, Italy.

Received: 27 June 2021 Accepted: 4 November 2021

Published online: 27 November 2021

\section{References}

1. Cornford P, Bellmunt J, Bolla M, Briers E, De Santis M, Gross T, et al. EAU-ESTRO-SIOG guidelines on prostate cancer. Part II: treatment of relapsing, metastatic, and castration-resistant prostate cancer. Eur Urol. 2017;71(4):630-42.

2. Giovacchini G, Picchio M, Briganti A, Cozzarini C, Scattoni V, Salonia A, et al. [11C]choline positron emission tomography/computerized tomography to restage prostate cancer cases with biochemical failure after radical prostatectomy and no disease evidence on conventional imaging. J Urol. 2010;184(3):938-43.

3. Giovacchini G, Picchio M, Coradeschi E, Bettinardi V, Gianolli L, Scattoni $V$, et al. Predictive factors of $[(11) C]$ choline PET/CT in patients with biochemical failure after radical prostatectomy. Eur J Nucl Med Mol Imaging. 2010;37(2):301-9.

4. Fuccio C, Castellucci P, Schiavina R, Guidalotti PL, Gavaruzzi G, Montini $\mathrm{GC}$, et al. Role of 11C-choline PET/CT in the re-staging of prostate cancer patients with biochemical relapse and negative results at bone scintigraphy. Eur J Radiol. 2012;81(8):e893-6.

5. Pasqualetti F, Panichi M, Sainato A, Matteucci F, Galli L, Cocuzza P, et al. [(18)F]Choline PET/CT and stereotactic body radiotherapy on treatment decision making of oligometastatic prostate cancer patients: preliminary results. Radiat Oncol. 2016;11:9.

6. Tosoian JJ, Gorin MA, Ross AE, Pienta KJ, Tran PT, Schaeffer EM. Oligometastatic prostate cancer: definitions, clinical outcomes, and treatment considerations. Nat Rev Urol. 2017;14(1):15-25.

7. Hellman S, Weichselbaum RR. Oligometastases. J Clin Oncol. 1995;13(1):8-10.

8. Reyes DK, Pienta KJ. The biology and treatment of oligometastatic cancer. Oncotarget. 2015;6(11):8491-524.

9. Pasqualetti F, Cocuzza P, Coraggio G, Ferrazza P, Derosa L, Galli L, et al. Long-term PSA control with repeated stereotactic body radiotherapy in a patient with oligometastatic castration-resistant prostate cancer. Oncol Res Treat. 2016;39(4):217-20.

10. Berkovic P, De Meerleer G, Delrue L, Lambert B, Fonteyne V, Lumen N, et al. Salvage stereotactic body radiotherapy for patients with limited prostate cancer metastases: deferring androgen deprivation therapy. Clin Genitourin Cancer. 2013;11(1):27-32.

11. Khoo V. Is there another bite of the cherry? The case for radical local therapy for oligometastatic disease in prostate cancer. Eur Urol. 2016;69(1):13-4.

12. Tree AC, Khoo VS, Eeles RA, Ahmed M, Dearnaley DP, Hawkins MA, et al. Stereotactic body radiotherapy for oligometastases. Lancet Oncol. 2013;14(1):e28-37.

13. Pasqualetti F, Panichi M, Sainato A, Baldaccini D, Cocuzza P, Gonnelli A, et al. Image-guided stereotactic body radiotherapy in metastatic prostate cancer. Anticancer Res. 2018;38(5):3119-22.

14. Slaoui A, Albisinni S, Aoun F, Assenmacher G, Al Hajj Obeid W, Diamand R, et al. A systematic review of contemporary management of oligometastatic prostate cancer: fighting a challenge or tilting at windmills? World J Urol. 2019;37(11):2343-53.

15. Gillies RJ, Kinahan PE, Hricak H. Radiomics: images are more than pictures, they are data. Radiology. 2016;278(2):563-77.

16. Sollini M, Bandera F, Kirienko M. Quantitative imaging biomarkers in nuclear medicine: from SUV to image mining studies. Highlights from annals of nuclear medicine 2018. Eur J Nucl Med Mol Imaging. 2019;46(13):2737-45.

17. Vilela RA, Navarro NF, Faria ET, Ferreira EB, Ruzza RZ, Gadia R, et al. Use of stereotactic body radiation therapy for oligometastatic recurrent prostate cancer: a systematic review. J Med Imaging Radiat Oncol. 2018;62(5):692-706.

18. Turpin A, Girard E, Baillet C, Pasquier D, Olivier J, Villers A, et al. Imaging for metastasis in prostate cancer: a review of the literature. Front Oncol. 2020;10:55.

19. Cornford P, van den Bergh RCN, Briers E, Van den BroeckT, Cumberbatch MG, De Santis M, et al. EAU-EANM-ESTRO-ESUR-SIOG Guidelines on Prostate Cancer. Part II-2020 update: treatment of relapsing and metastatic prostate cancer. Eur Urol. 2021;79(2):263-82.

20. Boellaard R, O'Doherty MJ, Weber WA, Mottaghy FM, Lonsdale MN, Stroobants SG, et al. FDG PET and PET/CT: EANM procedure guidelines for tumour PET imaging: version 1.0. Eur J Nucl Med Mol Imaging. 2010;37(1):181-200.

21. Boellaard R, Delgado-Bolton R, Oyen WJ, Giammarile F, Tatsch K, Eschner W, et al. FDG PET/CT: EANM procedure guidelines for tumour imaging: version 2.0. Eur J Nucl Med Mol Imaging. 2015;42(2):328-54.

22. UICC. TNM Classification of malignant tumours. In: Eight Edit. 2016. p. 688

23. Pasqualetti F, Panichi M, Sollini M, Sainato A, Galli L, Morganti R, et al. $\left[{ }^{18} \mathrm{~F}\right]$ Fluorocholine PET/CT-guided stereotactic body radiotherapy in patients with recurrent oligometastatic prostate cancer. Eur J Nucl Med Mol Imaging. 2020;47(1):185-91.

24. Gandaglia G, Fossati N, Stabile A, Bandini M, Rigatti P, Montorsi F, et al. Radical prostatectomy in men with oligometastatic prostate cancer: results of a single-institution series with long-term follow-up. Eur Urol. 2017;72(2):289-92.

25. Nioche C, Orlhac F, Boughdad S, Reuzé S, Goya-Outi J, Robert C, et al. LIFEx: a freeware for radiomic feature calculation in multimodality imaging to accelerate advances in the characterization of tumor heterogeneity. Cancer Res. 2018;78(16):4786-9. 
26. Zwanenburg A, Vallières M, Abdalah MA, Aerts HJWL, Andrearczyk V, Apte A, et al. The image biomarker standardization initiative: standardized quantitative radiomics for high-throughput image-based phenotyping. Radiology. 2020;295(2):328-38.

27. Sollini M, Kirienko M, Cavinato L, Ricci F, Biroli M, leva F, et al. Methodological framework for radiomics applications in Hodgkin's lymphoma. Eur J Hybrid Imaging. 2020;4(1):9.

28. Marín de Mas I, Aguilar E, Zodda E, Balcells C, Marin S, Dallmann G, et al. Model-driven discovery of long-chain fatty acid metabolic reprogramming in heterogeneous prostate cancer cells. PLOS Comput Biol. 2018;14(1):e1005914.

29. Balaban S, Nassar ZD, Zhang AY, Hosseini-Beheshti E, Centenera MM, Schreuder M, et al. Extracellular fatty acids are the major contributor to lipid synthesis in prostate cancer. Mol Cancer Res. 2019;17(4):949.

30. Peitzsch C, Gorodetska I, Klusa D, Shi Q, Alves TC, Pantel K, et al. Metabolic regulation of prostate cancer heterogeneity and plasticity. Semin Cancer Biol. 2020.
31. Wang Y, Ma S, Ruzzo WL. Spatial modeling of prostate cancer metabolic gene expression reveals extensive heterogeneity and selective vulnerabilities. Sci Rep. 2020;10(1):3490.

32. Gelardi F, Kirienko M, Sollini M. Climbing the steps of the evidence-based medicine pyramid: highlights from Annals of Nuclear Medicine 2019. Eur J Nucl Med Mol Imaging. 2020.

33. Ninatti G, Kirienko M, Neri E, Sollini M, Chiti A. Imaging-based prediction of molecular therapy targets in NSCLC by radiogenomics and AI approaches: a systematic review. Diagnostics (Basel). 2020;10(6).

34. Sollini M, Bartoli F, Marciano A, Zanca R, Slart RHJA, Erba PA. Artificial intelligence and hybrid imaging: the best match for personalized medicine in oncology. Eur J Hybrid Imaging. 2020;4(1):24.

\section{Publisher's Note}

Springer Nature remains neutral with regard to jurisdictional claims in published maps and institutional affiliations.

\section{Submit your manuscript to a SpringerOpen ${ }^{\circ}$ journal and benefit from:}

- Convenient online submission

- Rigorous peer review

- Open access: articles freely available online

- High visibility within the field

- Retaining the copyright to your article

Submit your next manuscript at $\gg$ springeropen.com 